\title{
Battling the opiate crisis: translating the latest advances in addiction biology into novel treatment strategies
}

\section{The opioid epidemic: a national health crisis}

The United States is experiencing an opiate addiction crisis that is unprecedented in modern times. As compared toyears prior to 1999, drug overdose deaths are increasing at an alarming rate in the United States. Over $60 \%$ of drug overdose deaths involve an opioid. From 2000 to 2015, more than a half million people died from drug overdoses, with the number of overdose deaths involving opioids quadrupling. ${ }^{1,2}$ Drug overdose deaths continue to increase in the United States. Approximately sixty percent of drug overdose deaths involve an opioid. ${ }^{1}$ We now know that overdoses from prescription opioids are a driving factor in the 15 year increase in opioid overdose deaths. ${ }^{2}$ This is a key piece of information as it points to a contributing factor that can be addressed: excessive and inappropriate prescribing of opiates. It also identifies the prescribing physician as an important locus for identification and intervention of opiate addiction. Coordination and cooperation between prescribing physicians and opiate abuse treatment providers is a necessary tool in stemming this crisis.

\section{The opioid epidemic: why is this happening?}

The magnitude and rapid progression of the opioid epidemic is a topic that must be addressed by the institutions that make social and economic policy. Many probable factors have set the conditions for this epidemic, including economic stress resulting from the "Great Recession" and the evolution of the economy from manufacturing to non-manufacturing industries, ${ }^{3}$ the fracturing of social and familial structures, ${ }^{3}$ a lack of hope for a better life, the decline of the middle class, and increasing income disparity. ${ }^{3,4}$ These data suggest that the current epidemic has been spurred by macroeconomic changes that have robbed many Americans of their financial status and security., It is not surprising that the current epidemic is most severe in those areas of the country that have lost the most economically as a result of the recession and the effects of globalization.

\section{Drug addiction and dependence}

Drug addiction is a chronic brain disease with strong genetic, neurodevelopmental, and sociocultural components. ${ }^{5,6}$ Drug addiction is characterized by drug-craving, drug-seeking behavior, and continuation of drug use despite adverse consequences to the user. An inter-related phenomenon, drug dependence, is a physiological state produced by repeated drug use and is characterized by observable withdrawal phenomena upon cessation of drug use. The two phenomena are inter-related due to drug withdrawal being a factor in continuing drug use to avoid physical drug withdrawal. Drug withdrawal phenomena are at the minimum unpleasant, and can be fatal, depending upon the drug.

\section{Neuropsychological components of drug addiction}

Key to understanding the neurobiology of drug addiction is an understanding of the neural processes contributing to the development of drug addiction. Among these neural processes are drug reward, drug
Volume 5 Issue 4 - 2017

\author{
BillW Massey \\ Chief Science Officer, Opiate Treatment Centers of America, \\ USA
}

Correspondence: Bill W Massey, Chief Science Officer, Opiate Treatment Centers of America, USA, Tel I 7604433782, Email bmassey@bwmassey.com

Received: August 16, 2017| Published: September 15, 2017

satiation, and drug-induced neuroplasticity resulting in diminished executive functioning. It has been known for some time that all drugs of abuse activate "reward" pathways in the brain. ${ }^{7}$ While not widely appreciated, it is becoming more apparent that drugs of abuse are affected by brain "satiation" mechanisms that are common across rewarding substances/actions (e.g. food) ${ }^{8}$ Another key element is the emerging data showing that almost all drugs of abuse produce impairment of executive functioning, contributing to making bad decisions and a lack of self-control. ${ }^{5}$ Each of these underlying neural processes provides a crucial point of intervention for the treatment of drug addiction. Also, the diagnosis and treatment of co-morbid psychiatric disorders is important for drug abuse treatment success, because drug abuse is often co-morbid with affective disorders and serious mental illness. ${ }^{9}$

\section{Initiation and progression of drug addiction}

To understand how initial drug "experimentation" progresses to drug addiction, it is important to understand the properties of abused drugs that lead to the drug-addicted state. All drugs of abuse exert their initial reinforcing effects by activating reward circuits in the brain. ${ }^{7}$ Continued drug use results in physiological changes to the brain (neuroplasticity) that interferes with the capacity to exert self-control over drug-taking behaviors and renders the brain more sensitive to stress and negative moods. ${ }^{5}$ While it is not completely understood why some individuals can experiment with drugs and not proceed down the path to drug addiction, it is known that individuals with genetic vulnerabilities, those exposed to chronic stress, or those suffering from comorbid psychiatric conditions are at greater risk of transitioning into the automatic and compulsive behaviors that characterize addiction. ${ }^{5-9}$

\section{Reward pathways and drug addiction}

The pleasant or "rewarding" effects of abused drugs underlie the motivation for initial and early subsequent drug-taking behavior. Key to understanding the biological basis for abused drug's rewarding effects is the finding that all drugs of abuse produce large increases in the release of dopamine in mesolimbic dopaminergic pathways associated with "reward", which result in their reinforcing effects. ${ }^{5-7}$ 
These supraphysiologic surges of dopamine in the mesolimbic structure known as the nucleus accumbens (Nac) activate the direct striatal pathway via dopamine type 1(D1) receptors and inhibit the indirect striatocortical pathway via dopamine type 2(D2) receptors. ${ }^{5}$ Each of these dopaminergic pathways contribute to the subjective "high" that is the basis for the rewarding properties of drugs. The greater and faster the drug-induced release of dopamine, the greater the subjective "high", which explains why smoking and intravenous injection are more rewarding and addictive than oral administration. ${ }^{5-7}$ There appear to be differences among abused drug classes as to the direct mechanism by which they produce the supraphysiologic surge in mesolimbic dopamine release. For example, opioid agonists induce mesolimbic dopamine release via decreasing GABAergic inhibition from medium spiny neurons on dopaminergic neuronal firing. ${ }^{10}$ Regardless of the specific mechanism; the stimulation of mesolimbic dopamine release in these reward circuits underlies the rewarding properties of abused drugs.

\section{Excessive midbrain dopamine activity leads to neuro plasticity and brain dysfunction, and the molecular mechanisms of drug-induced neuro plasticity}

So how does one get from the rewarding "high" to the malicious alteration of brain function that underlies the compulsive drug use that characterizes drug addiction? The answer lies in the neural basis of memory formation, a process called long-term potentiation. Long-term potentiation is modulated via glutamatergic mechanisms on larger synapses and dendritic spines. ${ }^{11}$ In long-term potentiation, repeated stimulation of neural circuits causes changes in the reactivity of neurons expressing the excitatory neurotransmitter glutamate. The increased reactivity of these neurons to glutamate is akin to strengthening a memory. These enhancements in reactivity are persistent, thus the name long-term potentiation. Drug-induced neuroplasticity evokes the same types of molecular processes involved in long-term potentiation and long-term depression that underlie learning and memory. ${ }^{11}$ Experimental data has demonstrated that the repeated exposure to supraphysiologic surges in dopamine release, such as produced by repeated drug administration, triggers these neuroplastic changes in glutamatergic inputs to the striatum and midbrain dopamine neurons, enhancing the brain's reactivity to drug cues, reducing the sensitivity to non-drug rewards, weakening self-control, and increasing the sensitivity to stressful stimuli and dysphoria. $^{5-12}$ These synaptic modifications generate a long-lasting molecular memory for the drug's rewarding and conditioning effects that influence subsequent behaviors. ${ }^{13}$

Also important to the remodeling of brain processes is the opposite of long-term potentiation, long-term depression. Long-term depression results in decreased reactivity of neurons and is modulated via GABAergic mechanisms on smaller synapses and dendritic spines. ${ }^{11}$ These neuroplastic changes in inhibitory GABAergic medium spiny neurons inhibit neural pathways projecting to the prefrontal cortex (PFC), resulting in cognitive impairments. ${ }^{5}$ Thus, drug-induced dopamine release results in strengthening or weakening (longterm depression) of synaptic connectivity in various brains reward regions. ${ }^{14}$ These neuroplastic changes depend upon the epigenetic enhancement or silencing of gene expression, such as expression of the transcription factor $\triangle \mathrm{FosB}$, and on post-transcription modulation of translation (RNA editing). ${ }^{15-18}$ Although drug-induced neuroplasticity starts in the brain areas processing reward, these neuroplastic changes eventually affect the entire brain and lead to dysfunctions of learning, executive functions, cognitive awareness and emotion..$^{5-11}$ In addition to neuroplastic changes affecting the mesolimbic reward pathways, neuroplastic changes in the frontal cortex play a key role in the compulsive and excessive drug use that is characteristic of drug addiction. Extensive research has shown that the neuroplastic changes in the mesolimbic reward pathways are directly implicated in the subsequent neuroplastic changes in the frontal cortex as the disease progresses. It has been shown that repeated drug exposures reduce levels of D2Rs in the striatum, including in the Nac. ${ }^{19}$ This is important since low levels of D2Rs in striatum are associated with impulsivity and predict escalating and compulsive drug self-administration. ${ }^{19}$ Human brain-imaging studies of addicted individuals have shown reductions in $\mathrm{D} 2 \mathrm{R}$ availability in the striatum for most abused drugs. ${ }^{20}$ Low levels of D2Rs in the striatum result in reduced DA inhibition of the indirect pathway, leading to reduced thalamo-cortical stimulation which results in reduced PFC activity, including reduced activity in the anterior cingulate (ACC) and orbitofrontal (OFC) cortices. ${ }^{20-22}$ The ACC and OFC are necessary for self-control and for processing salience attribution, and their disruption is associated with a propensity for impulsive and compulsive behaviors. ${ }^{22}$ Thus, low levels of D2R in striatum may increase the risk for compulsive drug taking in part by impairing PFC regions that inhibit prepotent responses and enable flexibility of behavioral choices as a function of changing environments. ${ }^{23}$ Figure 1 is a Schematic representation of the indirect pathways in which DA neurons from the ventral tegmental area (VTA) and the substantia nigra compacta (SN) provide DA inputs to the striatal GABA neurons expressing D2R (D2R-MSNs). These striatal neurons target GABA cells in the globus pallidum external (GPe), which provide inhibition to glutamatergic neurons within the subthalamic nucleus (STN). The STN glutamatergic neurons provide an excitatory input to GABA neurons present in the substantia nigra reticulata (SNR) and the globus pallidum internal (Gpi), which inhibit glutamate neurons of the thalamus innervating the frontal cortex. Drug-induced reductions in D2R within the striatum impair the inhibition of this indirect pathway by DA, resulting in reduced thalamo-cortical stimulation and consequently reduced activity in the frontal cortex. Relationship between D2R in striatum and brain glucose metabolism in frontal regions of drug abusers tested both with $[11 \mathrm{C}]$ raclopride and FDG. Images next to the y axis show axial brain metabolic images at the level of the orbitofrontal cortex, and images below the $\mathrm{x}$ axis show axial images of $\mathrm{D} 2 \mathrm{R}$ striatal availability for a control and a cocaine abuser. Regression slopes correspond to the association between metabolism in the orbitofrontal cortex (OFC) and D2R availability in striatum in cocaine-addicted and in methamphetamine- addicted subjects.

\section{The neuroplastic reductions in cortical D2 receptors are at least semi-permanent}

The above-described deficits in D2 receptors in human drug abusers persist even after prolonged periods of abstinence. This persistence in D2 receptor deficits suggests that these altered levels are not solely drug-induced. ${ }^{24}$ Data from genetic studies suggest an important role for genetic factors that affect D2 receptor availability and may predict inter-individual responses to drugs of abuse. ${ }^{25}$ For example, there are known mutations of the gene that encodes the D2 receptor, DRD2, that are linked to decreased expression of the D2 receptor, thereby decreasing their number. It has been shown that these DRD2 mutation-produced D2 receptor deficiencies can 
exacerbate drug-induced D2 receptor abnormalities and predispose a patient to drug addiction. ${ }^{25}$

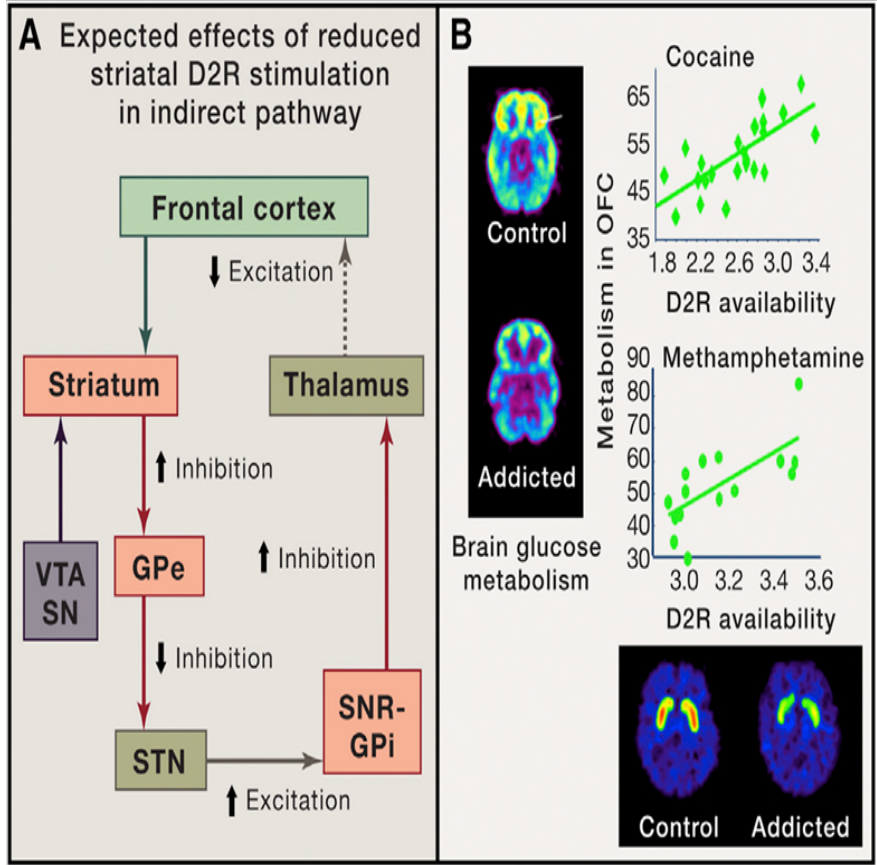

Figure I A, Indirect pathways in which DA neurons from the ventral tegmenta area (VTA) and the substantia nigra compacta (SN) provide DA inputs to the striatal GABA neurons expressing D2R (D2R-MSNs); B, Relationship between $D 2 R$ in striatum and brain glucose metabolism in frontal regions of drus abusers tested both with [IIC] raclopride and FDG.

\section{Neuroplasticity and tolerance:A downward spiral}

These dopamine-evoked neuroplastic changes are likely to contribute to the emergence of tolerance to the drug's effects. Tolerance prompts the need to use increasingly larger doses in an attempt to achieve the same effect. ${ }^{7}$ In turn, exposure to higher drug doses that further increases dopamine release also facilitates the neuroplastic changes that ultimately change the reactivity of brain dopamine pathways to drugs and drug cues. ${ }^{5}$ This vicious cycle of increased tolerance, increased drug doses, and the neuroplastic changes they engender further perpetuate the downward spiral of increased consumption and associated severity of the addiction and drug dependence. ${ }^{25}$

\section{BDNF, neuroplasticity, and addiction risk}

Brain-derived neurotrophic factor (BDNF) is a neurotrophic factor implicated in many critical neurological processes such as cell survival, proliferation, synaptic growth through development, and hippocampal changes associated with learning and conditioned drug reward. ${ }^{26}$ Because of its impact on synaptic formation and the relation of synaptic remodeling in the progression of drug addiction, it is not surprising that BDNF may play a role in the neuroplasticity underlying the processes that lead to drug addiction. Interestingly, plasma BDNF levels are significantly higher in abstinent methamphetamine abusers compared to controls and in multiple substance abusers with schizophrenia. ${ }^{27,28}$ Recent association studies have linked an increased incidence of the purported functional BDNF gene polymorphism val66met and substance abuse in males. ${ }^{29,30}$ This mutation appears to affect declarative memory in humans. ${ }^{31}$ Recent work on the cognitive impairments associated with schizophrenia suggest that schizophrenia produces its cognitive impairments via mechanisms in the prefrontal cortex, which results in decreased activity, impaired declarative memory, and diminished executive function. ${ }^{32-34}$ Perhaps, there is a linkage between the disproportionate number of schizophrenics that abuse drugs when compared to the general population (7-fold greater) and diminished executive function that is shared by both schizophrenics and non-schizophrenic drug abusers.

\section{OPRMI and addiction risk}

OPRM1 is the gene that encodes the $\mu$ opiate receptor. The $\mu$ opiate receptor is the site where opiates produce their characteristic pharmacological effects such as analgesia, sedation, slightly reduced blood pressure, itching, nausea, euphoria, respiratory depression, miosis, and decreased bowel motility leading to constipation. ${ }^{35}$ The A118G polymorphism in the OPRM1 gene results in a decrease in expression of $\mu$ opiate receptors and decreased functionality of the expressed receptors, which may decrease the analgesic response to opiates. Patients with this variant have shown a lower pain threshold and a higher drug consumption to achieve effective analgesia. Because of its functional significance, this variant (rs1799971, A118G, Asn40Asp) in OPRM1 has been extensively studied in addiction. ${ }^{35,36}$ A collaborative meta-analyses of 25 data-sets with over 28,000 European-ancestry subjects investigated this OPRM1 mutation for non-specific risk for "general" substance dependence. ${ }^{36}$ The study compared cases dependent on any substance to controls that were non-dependent on all assessed substances: alcohol, opioid, cannabis, and cocaine dependence, and nicotine. ${ }^{36}$ The $G$ allele showed a modest protective effect on general substance dependence $(\mathrm{OR}=0.90$, $95 \%$ C.I. [0.83-0.97], p value $=0.0095, \mathrm{~N}=16,908)$. Results were similar for each drug or drug class examined. ${ }^{36}$ These results show that rs1799971 contributes to mechanisms of addiction liability that are shared across different addictive substances. ${ }^{36}$ Paradoxically, this same mutation results in an apparent gain of function with respect to response to endogenous opioids, like $\beta$-endorphins. Individuals who carry at least $1 \mathrm{G}$ allele have significantly better outcomes with naltrexone therapy for alcoholism and drug abuse. ${ }^{37}$

\section{Why do oprmI polymorphisms affect susceptibility to abused drugs across drug classes?: opioid regulation of the mesolimbic da pathway}

The endogenous opiate system is intricately involved in the activity of the mesolimbic dopamine reward pathway. A key component of this involvement is that $\mu$ opiate receptor agonism increases the sensitivity of the mesolimbic dopaminergic reward pathway. ${ }^{38}$ Because of this effect on the mesolimbic reward pathway, functionality of the $\mu$ opiate receptor, and thus, mutations in OPRM1 that may affect functionality, is involved in the reinforcing effects of drugs across classes of abused drugs. The OPRM1 A118G mutation decreases the ability of mu receptor agonism to increase dopamine release in the reward pathways. ${ }^{38}$ Thus, one would surmise that individuals that carry this mutation would not get as much reward from an abused drug. While this may be true for initial use, this limitation can be overcome by increased doses of the abused drug, which, in turn, can accelerate the neuroplastic changes that result in addiction. If the user was persistent in their attempts to get "high", the higher doses needed could explain the increased risk of drug addiction associated with 
this mutation. Unfortunately, this same mutation, while decreasing $\mathrm{mu}$ opiate receptor activity, also increases risk of death from opiate overdose 91-fold. ${ }^{39}$ This increased risk of overdose is postulated to be a result of having to take increased opiate doses to produce an effect, differential development of tolerance to different opiate effects (e.g. faster tolerance development to euphoria versus respiratory depression), and the use of very high-affinity opiates (e.g. heroin). ${ }^{39}$ This mutation-induced effect may explain the high risk of overdose when users of lower-affinity opiate users (e.g. oxycodone) switch to high-affinity opiates (e.g. fentanyl or heroin).

\section{Endogenous opioids and addiction}

Basic research has shown that the endogenous opiate system plays a crucial role in addiction biology across drug classes. This involvement is primarily focused on modulation of DA reward processes and in the production of anhedonic withdrawal states upon drug abstinence. In general, the enkephalins are associated with pleasurable stimuli, whereas the dynorphins are associated with dysphoric stimuli, especially after neuro-adaptation to chronic drug use, and may underlie some of the dysphoric aspects of opiate withdrawal. Endogenous opioids modulate DA neuronal firing in midbrain and striatal MSNs, where dynorphin co-localizes in D1RMSNs and enkephalin in D2R-MSNs. ${ }^{40-42}$ This dual effect on D1 and D2 expressing MSNs indicates an effect of endogenous opiates in the direct and indirect reward pathways. Endogenous opioids, especially enkephalins, are implicated in hedonic responses to natural and drug rewards and in the adaptations that follow repeated drug exposures and drug relapse. ${ }^{43,44}$ Genetic susceptibility to drug abuse is supported by the finding that drug-induced increases in enkephalins are enhanced in patients that are homozygous for the OPRM1 A118G mutation (AA). ${ }^{45}$

The physical and mental discomfort that accompanies drug withdrawal can serve as an impetus for continued drug use. The kappa opiate receptor and its endogenous agonist, dynorphin, may be an important target for therapeutic intervention after drug cessation, and particularly, in the withdrawal period. The endogenous opioid, dynorphin, through its activation of kappa receptors (KOR), is implicated in the stress-induced potentiation of drug reward, and thus, may facilitate relapse by enhancing drug reward during periods of distress, such as drug withdrawal. ${ }^{46}$ Conversely, activation of KOR on DA terminals inhibits DA release in the NAc, which is implicated in the dysphoria that follows drug withdrawal. ${ }^{47} \mathrm{KOR}$-mediated inhibition of DA release may decrease the reinforcing properties of drugs and may help to prevent relapse in patients that have recovered from withdrawal. These findings have generated interest in KOR antagonists or partial agonists as medications to prevent relapse in addiction. ${ }^{48}$

\section{The need for an interdisciplinary approach to an unprecedented crisis}

There is widespread agreement that current treatment strategies are not adequate to address the opiate abuse crisis. Current treatment methodologies are outdated and ineffective for the vast majority of patients. The possible reasons for the high recidivism rates seen with these traditional drug abuse treatment programs are myriad: self-defeatism, reliance on a "higher-being", artificial environment, non-medical viewpoint, addiction not treated as a disease but rather as a moral failing, etc. Recent discoveries in the biological basis for addiction point to new therapeutic approaches, many of which have been shown effective in structured clinical research. The time is now to introduce these recent findings in the neuroscience of addiction into clinical practice. A new treatment paradigm is needed that integrates new findings in genetics, pharmacogenetics, the biology of addiction, neurobehavioral research, and treatment of co-morbid psychiatric disorders.

\section{Genetic biomarkers of addiction risk and severity}

Recent advances in genetics and molecular biology have provided insight into underlying biological susceptibility to drug addiction. Foremost has been the discovery of genetic variants predictive of predisposition for addiction and severity of addiction outcomes, which usually affect brain reward and/or satiation pathways. By instituting genetic testing into the addiction treatment paradigm, one can identify genetic variants that have neurobehavioral implications (e.g. impulsivity) that are useful in customizing treatment to the individual patient. The following genetic tests have direct clinical applicability:

DRD2 polymorphisms: These mutations result in decreased expression and function of D2 receptors. D2 receptors are involved in brain reward systems and underlie drug-induced cognitive impairments. Certain variants of this gene may lead to increased consumptive behaviors such as over-eating and increased propensity for drug abuse. ${ }^{24,25}$

BDNF Val66Met polymorphism: This mutation results in decreased BDNF expression and function. BDNF affects the neurodevelopment of the mesolimbic dopamine pathways involved in reward systems. ${ }^{26-34}$

OPRM1 A118G polymorphism: Activation of the $\mu$ receptor by an agonist such as morphine causes analgesia, sedation, slightly reduced blood pressure, itching, nausea, euphoria, respiratory depression, miosis, and decreased bowel motility leading to constipation. The A118G polymorphism in the OPRM1 gene results in a decrease in expression of $\mu$ opiate receptors, which may decrease the analgesic response to opiates. Patients with this variant have shown a lower pain threshold and a higher drug consumption to achieve effective analgesia. This mutation is also associated with a much greater risk of opiate overdose. Patients with this mutation need more intensive neurobehavioral intervention and supportive measures due to the enhanced overdose risk. Paradoxically, this same mutation results in an apparent gain of function with respect to response to endogenous opioids, like $\beta$-endorphins. Individuals who carry at least $1 \mathrm{G}$ allele have significantly better outcomes with naltrexone therapy for alcoholism and drug abuse. ${ }^{35-39}$

MTHFR polymorphisms: The MTHFR gene codes for an enzyme called methylenetetrahydrofolate reductase. Methylenetetrahydrofolate reductase converts the B-vitamin folate to 5-methyltetrahydrofolate, the active form that can get into the brain. Methyl-folate is involved in the synthesis of the neurotransmitter serotonin. Serotonin suppresses mesolimbic dopamine release and can impact the functioning of the reward system. MTHFR impairment presents an increased risk of depression, drug abuse, and may predispose patients to be resistant to medical treatment.

\section{The neurobehavioral continuum of the pro- cess of addiction}

Advances in addiction biology indicate that two separate, but intertwined, neural processes are at the heart of the transformation 
of initial drug use into drug addiction. Each consists of two opposing components, with the predominance of one being a driver of addiction. These processes are Reward versus Satiation and Executive Control versus Impulsivity. Reward versus Satiation is critically important for initial use. Executive Control versus Impulsivity comes into play with the brain changes that accompany continued use. These are pictured below as opposing "ying/yang" processes (Figure 2). The neurobehavioral continuum is diagrammatically presented below Figure 3 .
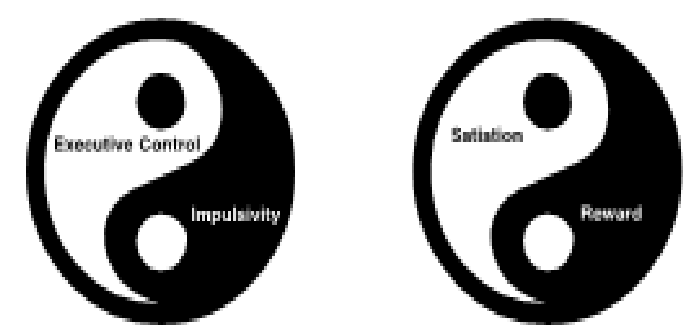

Figure 2 Opposing "ying/yang" processes.

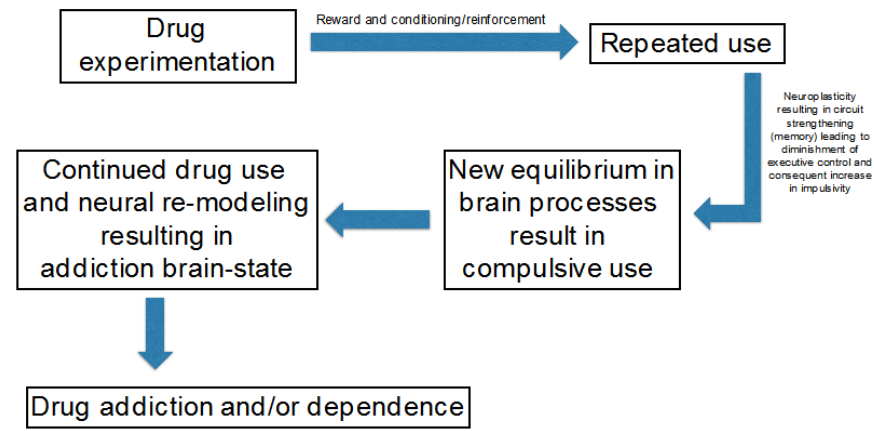

Figure 3 Neurobehavioral continuum of the process of addiction.

\section{Translating advances in addiction science into clinical practice: targets for therapy}

Behavioral neuroscience findings across multiple disciplines indicate that drug addiction should be treated in an interdisciplinary manner. Drug addiction is a chronic brain disease that requires multimodal therapeutics customized to a patient's genetics and clinical presentation. Drug abuse treatment should address psychiatric treatment of psychiatric co-morbidities. New approaches involve manipulation of the reward-satiation relationship, manipulation of the executive control-impulsivity relationship, Pharmacotherapeutic management of reward, satiation, and withdrawal until new equilibriums are established. Pharmacologic therapeutics include replacement therapies for opiates, dopamine modulating agents, and 5-HT2C receptor agonism.

\section{Reward and satiation: interacting processes that play a pivotal role in initiation and conti- nuance of drug abuse}

All drugs that are abused produce pleasurable or euphoric psychological states. Subjective attestation of this property of abused drugs can be obtained from anyone that has ever used an abused drug. From a research perspective, it has been shown that drugs of abuse can act as a reward for performing a trained (i.e. conditioned) response. For example, a drug of abuse can act very much the same way that a "treat" does when training a dog to sit. Presumably, this reward aspect of abused drugs is because of the pleasurable psychological state they produce. In the experimental analysis of behavior, drugs are said to serve as a reinforcer of conditioned behavior, in that the presentation of a reinforce increases the probability of the behavior that precedes it. In behavioral parlance, drugs that can serve as a reward are called reinforcers. No clearer example of experimental drug-taking behavior can be found than drug self-administration experiments. In these experiments, animals are implanted with intravenous lines that can deliver drugs directly into their blood stream, and drugs are delivered once the animal has performed the response (e.g. lever press). Almost all drugs that are used by humans for their psychoactive effects are reinforcers (rewarding) in animal operant self-administration experiments, with the exceptions being serotonergic hallucinogens and cannabis (these drug classes are hard to establish as reinforcers). Neurobehavioral research has clearly shown that the reinforcing effects of abused drugs are produced by their ability to increase the release of dopamine in the mesolimbic reward pathway, and that all drugs abused by humans, regardless of drug class, increase dopamine release in the mesolimbic reward pathway. While the neurobiological basis for drug reinforcement is dependent upon dopamine release in the mesolimbic reward pathway, there are many external (i.e. environmental) events that can have an impact of drug reinforcement. For example, the external environment that is associated with drug-taking can become a key component of the reinforcing aspects of drugs. Such concurrent environmental stimuli can become conditioned with the drug experience and can have a strong influence on drug-taking behavior.

The strength of reinforcement is dependent upon internal and environmental conditions, such as the state of satiation or environmental context. As such, there is a relationship between satiation and reinforcement (reward). There is abundant evidence that common systems regulate these processes (common reward pathways and common satiation pathways). The evidence for a common reward pathway has been discussed earlier in this manuscript. The evidence for common satiation pathways, while not as explored or robust as the evidence for a common reward pathway, is still quite convincing despite the relative lack of attention it has received.

The existence of a common satiation pathway for reinforcers is supported by a phenomenon known as the food deprivation effect. When deprived of food, animals will self-administer much greater amounts of drugs of abuse. ${ }^{49}$ Different classes of reinforcers can substitute for each other in a manner that indicates a common level of collective satiation exists, and by deduction, a common satiation pathway in the brain underlies this phenomenon. Many operant choice experiments, in which the animal can choose between drug self-administration and some other non-drug reinforce (e.g. food), have shown that concurrent availability of other non-drug reinforcers decreases drug choice and consumption. ${ }^{50}$ This laboratory phenomenon also is congruent with sociological studies of drug abuse that show higher rates of drug addiction in lower socioeconomic classes. The common satiation pathway explanation for the discordant rate of addiction in lower socioeconomic classes is that drug use is an easy to substitute reinforcer in a situation where non-drug reinforcers are not available. The current experimental evidence indicates that the brain's satiation pathway involves the ventromedial hypothalamus and 5-HT2C receptors. The involvement of the ventromedial hypothalamus is demonstrated 
by studies in which lesioning of the ventromedial hypothalamus by injection of gold thioglucose produces a hyperphagia of palatable foods and resultant obesity. ${ }^{51}$ In other words, the animals acted as if they could not be satiated after their ventromedial hypothalamus was destroyed. Interestingly, access to non-palatable food does not induce the food deprivation effect. This would indicate that the effect is not due to enhancement of mastication-related motor effects, but rather, is dependent upon the reward value of the concurrently available food. This palatability requirement further demonstrates a linkage between reward and satiation. While it has been demonstrated that the ventromedial hypothalamus is involved in satiation from reinforcers, it is in understanding the biochemical underpinnings that offer the most promise in finding a pharmaceutical solution for satiation. It has been well-explored that 5-HT2C receptor agonism reduces food intake and drug self-administration via an effect on satiation..$^{52,53}$ In fact, lorcaserin is a 5-HT2C agonist approved by FDA for obesity and (surprise!) has been shown to decrease drug self-administration. ${ }^{2}$ As such, lorcaserin may have utility in the off-label treatment of drug abuse and in improving cognitive function in addiction patients. ${ }^{53-55}$ As we will describe later, drugs that improve cognitive function may also play a role in fighting drug abuse by increasing executive control over impulsive behavior.

\section{Translating advances in addiction science into clinical practice: reward and satiation}

Substitution of healthy non-drug reinforcers to reduce drug consumption: The effects of concurrently available reinforcers on reducing drug self-administration suggest that the provision of non-drug reinforcers that increase mesolimbic dopamine release should decrease drug consumption and/or craving. In fact, that is the case. Moderate- to high-intensity exercise during the initial period of abstinence reduces relapse presumably via increased dopamine release in mesolimbic dopaminergic tracts and decreased glutamatergic activity. ${ }^{56}$ Additionally, there is evidence that exercise can induce BDNF expression and facilitate neuronal remodeling and normalized activity in mesolimbic dopaminergic neuronal pathways. ${ }^{56}$ The induction of BDNF and increased neural remodeling may offer support to long-term drug abstinence. The timing of initiation of exercise therapy is critically important. Exercise is useful when initiated just after drug cessation. However, exercise may increase relapse risk in patients that have been abstinent for some time (more than 2 weeks).$^{56}$ This differential response may be because when exercise is initiated right after drug abstinence, it serves as a substitute reinforce and contributes to satiation and reduces drug craving. The opposite effect seen when exercise is initiated later may be due to the existence of a time window for when substitution can be effective and reduce drug craving. When dopamine efflux is increased by initiating exercise at a later time following drug cessation, instead of contributing to overall satiation, the dopamine efflux triggers a "memory" of the drug state and instigates drug craving. Like exercise, exposure to music of the patient's choice is associated with increased mesolimbic dopamine release, induces BDNF, and normalizes activity of mesolimbic dopaminergic pathways. ${ }^{56}$ Music therapy has been shown to improve symptoms in Parkinson's disease, another disease linked to dopamine abnormalities..$^{57,58}$ Also like exercise, initiation of music therapy should be limited to the initial period of abstinence (within 2 weeks of cessation of drug use).$^{56}$ It follows that providing multiple non-drug concurrent reinforcers may decrease drug use. Increased availability of reinforcing social events, complementing desirable behaviors, and involvement in team activities may provide non-drug alternative reinforcement.

\section{Executive control vs. impulsivity: Competing neuro- behavioral decision systems (CNDS)}

Addiction research applying economic models (i.e. behavioral economics) has led to findings that imply a state of disequilibrium between two competing systems underlying decision making play a strong role in drug addiction. CNDS posits that the neural processes underlying executive control become weakened by brain changes that occur resultant to chronic drug use. Thus, addiction is a state of pathological overvaluation of drug reinforcers produced by imbalances in competing neurobehavioral decision systems (the executive and the impulsive/hedonic). ${ }^{59}$ This weakening of executive control (frontal cortex) results in a disinhibition of neural processes underlying impulsivity (mid-brain) and leads increased compulsive drug-taking behavior.

\section{Translating advances in addiction science into clinical practice: executive control and impulsivity}

\section{Strategies to reduce drug demand}

Addiction treatments that increase the short-term cost of drug use (e.g. contingency management), and drug agonist replacement therapies that decrease the short-term value of drug use, are backed by empirical evidence in both animal models and clinical interventions. ${ }^{59}$ Contingency management strategies can increase the price of drug use by adding an immediate incentive for abstinence, which is forfeited by drug use (e.g. money, vouchers, prizes). The repeated offering of abstinence-contingent reinforcers results in the devaluation of drug reinforcers and the increased valuation of non-drug reinforcers. ${ }^{60}$ Cost-focused motivational interventions can increase the saliency of the consequences of drug use by emphasizing the opportunity costs of drug use and shift value towards non-drug reinforcers (i.e. shifting relative control from the impulsive to the executive decisionmaking system). ${ }^{59}$ When two reinforcers are concurrently available, manipulating the relative cost differential between the two reinforcers can shift choice to the lower cost reinforcer. This is most effective when the reinforcers are of similar strength and are substitutable (e.g. gourmet food, chocolate, thrilling activities, music, sociallyrewarding experiences). ${ }^{59} \mathrm{~A}$ community reinforcement approach is an example of a cross-price demand application. Introducing a sociallyengaging activity such as participation in a community sports league can decrease impulsivity (discouraging drug use) while increasing executive control by nurturing prosocial behaviors. ${ }^{59}$ Experimental data predicts that interventions that enhance executive control will be more efficacious than those that aim to diminish impulsive control. Examples of interventions that increase executive control include interventions that enhance delayed-gratification ability and introducing alternative positive reinforcers, which have a greater effect than punishment of drug use..$^{59,60}$ Drug agonist therapies (i.e. drug replacement) can also decrease drug demand since provision of an alternative drug reinforcer substitutes for the detrimental drug and decreases demand for other detrimental drug reinforcers. ${ }^{59}$ Transcranial Magnetic Stimulation (TMS) may also improve executive function. Repeated administration of dIPFC TMS may reduce demand across reinforcers, and thus, increase relative tone of executive control. ${ }^{59-61}$

\section{Strategies related to delay discounting}

Delay discounting is a term psychologists use to describe the human tendency to favor tangible short-term (immediate) rewards 
over potentially greater long-term rewards (e.g. choosing the shortterm reinforcement of drugs over the much greater in magnitude long-term reinforcers that come from drug abstinence). Thus, delay discounting shows the contribution of the immediacy of reinforcement versus the magnitude of reinforcement. High rates of discounting are associated with drug abuse. Working memory training and resultant improvements in working memory increase executive control and decrease delay discounting. ${ }^{59}$ FMRI studies have shown increases in frontal cortex neural plasticity following working memory training. ${ }^{62}$ The nootropic agent modafinil has been shown to decrease delay discounting in alcoholic subjects ${ }^{63}$ and may increase executive control. $^{59}$

\section{Toxicology testing in drug abuse treatment}

Toxicology testing, a crucial component of treatment, can provide important information to the addiction treating physician. Toxicology testing provides an objective assessment of program compliance and is a key outcome metric. ${ }^{64,65}$ It enables a physician to know which drugs (licit and illicit) the patient is using and whether these have a negative or positive impact on drug abuse treatment. ${ }^{65}$ For instance, a positive test for heroin use would have a negative impact on the patient's recovery, whereas a positive test for buprenorphine when prescribed as part of treatment would indicate patient compliance. Due to the adverse sequela from reporting a false positive, a toxicology testing program must incorporate confirmatory testing by quantitative analytical method for all initial positive tests (e.g. LC-MS). ${ }^{64,65}$ Since the treating physician will want to know how the patient is progressing on an ongoing basis, longitudinal testing is required to effectively monitor compliance and treatment progress. ${ }^{65}$ A positive test result can be used to identify additional treatment needs and intensity of therapy ${ }^{65}$ Since drug metabolism is greatly affected by genetics, it is imperative that toxicology testing be used in conjunction with PGx. PGx testing can identify metabolic abnormalities that may confuse interpretation of results. ${ }^{64}$

\section{A framework for optimal medically-based treatment of opiate abuse}

The treatment of addiction in the US is failing: both to the patients and to society. There are numerous reasons for this failure. Almost none of the advances in addiction science have been translated into clinical practice. Insurance reimbursement is uncertain for addiction treatment and the costs of traditional treatment center-based treatment are unaffordable for patients. The treatment paradigms of the " 12 step programs" are unscientific and have an overwhelming failure rate. One reason that residential treatment centers fail is because such facilities create an artificial environment that is unrelated to the outside world. Once a patient leaves the facility they are back into the environment where drugs are available, environmental stimuli provoke urges to use drugs (conditioned reinforcers), and therapy is discontinued. It is no wonder as to why traditional addiction treatment is failing. The opiate crisis warrants an emergency response. The best approach to responding to this crisis is to incorporate and implement the latest findings from addiction science, treat addiction as a chronic condition, provide pharmaceutical therapies to reduce relapse and improve functioning, use the latest molecular diagnostics, and provide non-pharmacological treatments and cognitive behavioral therapy to directly combat the pathophysiology of addiction. In short, we should leverage scientific advances in addiction medicine in the treatment of opiate addiction by translating them into its treatment paradigm.

\section{Key components of an optimal medically-ba- sed treatment program}

\author{
a. Out-Patient Setting. \\ b. Affordable (Cash and Insurance). \\ c. Consideration of Co-Morbidities.
}

d. Buprenorphine-Naloxone Pharmacotherapy.

e. Medical Management Counseling.

f. Integrated Pharmacogenetics \& Toxicological Testing Program.

g. Behavioral Economic- \& Neuro economic-Based Therapeutic Approaches.

h. Translation of the latest scientific advances from addiction research into clinical practice.

\section{i. Longitudinal treatment reflecting the chronic nature of addiction.}

Such an approach directly addresses the causes of traditional treatment failure. This treatment paradigm can be done on an outpatient basis, allowing the patient to overcome their addiction in their daily environment and allowing them to continue to fulfill their societal roles as mothers, fathers, and employees. Out-patient treatment allows the patient to address their disease in their natural environment, exposed to the same stimuli as when they were abusing, making their recovery more effective and meaningful to their lives. It is also critical to address other psychiatric co-morbidities that may contribute to addictive behavior. Most importantly, this treatment paradigm is a fraction of the cost of traditional residential treatment. Therefore, even if insurance reimbursement is lacking, the patient can afford to continue their treatment. Affordability increases treatment access to patients that otherwise would be unable to get help. Treatment facilities should be non-descript, allowing patients to obtain treatment discretely, and thus, avoiding any embarrassment due to the misplaced societal stigma surrounding addiction. Upon intake, the patient should undergo a physical examination to assess overall health status, medical history, and to acquire samples for diagnostic tests (e.g. genetic testing, blood chemistry, toxicology, etc.). Based upon the patient's diagnostic results, a treatment program can be designed around the best treatment modalities to address each patient's unique biology and needs. To assist in management of withdrawal and help prevent relapse, buprenorphine-naloxone pharmacotherapy and medical management visits should be the basis of any medically-based treatment, in the absence of other factors to the contrary. Additional treatment modalities can be administered according to the physician's recommendations and the patient's choices.

During the initial phase of treatment, the patient should be intensively supervised (including a 1-2 day inpatient stay if warranted) and made ready to begin buprenorphine-naloxone pharmacotherapy. Also during the initial phases of therapy, psychiatric co-morbidities should be assessed and treatment recommendations provided that is based upon their genetic and pharmacogenetics profile. The patient should be scheduled for treatment appointments as needed based upon the treatment program and treatment modalities chosen. It is imperative that the patient be monitored for relapse and treatment compliance through regularly-administered toxicology testing for drugs and drug metabolites. Toxicology testing also provides an objective assessment of treatment success. The emergency nature of the present opiate crisis makes it imperative that the success of treatments be assessed. The 
results of objective assessment of treatment outcomes are necessary to make improvements in the treatment of opiate addiction.

\section{Acknowledgements}

None.

\section{Conflict of interest}

Author declares that there is no conflict of interest.

\section{References}

1. Rudd RA, Seth P, David F, et al. Increases in Drug and Opioid-Involved Overdose Deaths-United States 2010-2015. MMWR Morb Mortal Wkly Rep. 2016;65(5051):1445-1452.

2. CDC Wonder: Centers for Disease control and Prevention. Atlanta, USA; 2016

3. Keyes KM, Magdalena C, Brady JE, et al. Understanding the rural-urban differences in nonmedical prescription opioid abuse in the United States. Am J Public Health. 2014;104(2):e52-e59.

4. Dombrowski K, Crawford D, Bilal Khan, et al. Current Rural Drug Use in the US Midwest. J Drug Abuse. 2016;2(3):22.

5. Volkow N, Morales M. The Brain on Drugs: From Reward to Addiction. Cell. 2015;162(4):712-725.

6. Jadidi N, Nakhaee N. Etiology of Drug Abuse: A Narrative Analysis. Journal of Addiction. 2014;35825:1-6.

7. Koob G, Moal M. Drug Addiction, Dysregulation of Reward, and Allostasis. Neuropsychopharmacology. 2001;24(2): 97-129.

8. Abdelghany S, Pauli M Lorcaserin. A novel, selective 5-HT2C agonist for the treatment of obesity. Formulary. 2010;45:180-186.

9. Regier DA, Farmer ME, Rae DS, et al. Comorbidity of Mental Disorders with Alcohol and other Drug Abuse. JAMA. 1990;264(19):2511-2518.

10. Paladini CA, Roeper J. Generating bursts (and pauses) in the dopamine midbrain neurons. Neuroscience. 2014;282:109-121.

11. De Roo M, Klauser P, Garcia PM, et al. Spine dynamics and synapse remodeling during LTP and memory processes. Prog Brain Res. 2008;169:199-207.

12. Trifillieff P, Martinez D. Imaging addiction: D2 receptors and dopamine signaling in the striatum as biomarkers for impulsivity. Neuropharmacology. 2014;76:498-509.

13. Hyman SE. Addiction: a disease of learning and memory. Am J Psychiatry. 2005;162(8):1414-1422.

14. Grueter B, Rothwell PE, Malenka RC. Integrating synaptic plasticity and striatal circuit function in addiction. Curr Opin Neurobiol. 2012;22(3):545-551.

15. Kenny P. Epigenetics, microRNA, and addiction. Dialogues Clin Neurosci. 2014;16(3):335-344.

16. Robison JA, Nestler E. Transcriptional and epigenetic mechanisms of addiction. Nat Rev Neurosci. 2011;12(11):623-637.

17. Satterlee JS, Basanta-Sanchez M, Blanco S, et al. Novel RNA modifications in the nervous system: form and function. $J$ Neurosci. 2014;34(46):15170-15177.

18. Maze I, Herbert EC, David MD, et al. Essential role of the histone methyltransferase G9a in cocaine-induced plasticity. Science. 2010;327(5962):213-216.
19. Everitt B, Belin D, Economidou D, et al. Neural mechanisms underlying the vulnerability to develop compulsive drug-seeking habits and addiction. Philos Trans R Soc Lond B Biol Sci. 2008;363(1507):3125-3135.

20. Volkow ND, Baler RD. Addiction science: Uncovering neurobiological complexity. Neuropharmacology. 2014;76:235-249.

21. Black K, Koller JM, Campbell MC, et al. Quantification of indirect pathway inhibition by the adenosine A2a antagonist SYN115 in Parkinson disease. J Neurosci. 2010;30(48):16284-16292.

22. Volkow ND, Fowler JS. Addiction, a disease of compulsion and drive: involvement of the orbitofrontal cortex. Cereb Cortex. 2000;10(3):318-325.

23. Volkow ND, Wang GJ, Begleiter H, et al. High levels of dopa mine D2 receptors in unaffected members of alcoholic families: possible protective factors. Arch Gen Psychiatry. 2006;63(9):999-1008.

24. Volkow ND, Fowler JS, Wang GJ, et al. Dopamine in drug abuse and addiction: results of imaging studies and treatment implications. Arch Neurol. 2007;64(11):1575-1579.

25. Trifillieff P, Ducrocq F, Van Der Veldt S, et al. Blunted dopamine transmission in addiction: Potential mechanisms and implications for behavior. Semin Nucl Med. 2017;47(1):64-74.

26. Russo SJ, Mazei-Robison MS, Ables JL, et al. Neurotrophic factors and structural plasticity in addiction. Neuropharmacology. 2009;56(Suppl 1):73-82.

27. Su H, Tao J, Zhang J, et al. An association between BDNF Val66Met polymorphism and impulsivity in methamphetamine abusers. Neurosci Lett. 2014;582:16-20.

28. Liu Q, Walther D, Tomas D, et al. Human brain-derived neurotrophic factor (BDNF) genes, slicing patterns, and assessments of associations with substance abuse and Parkinson's disease. AM J Med Genetics. 2005;134(1):93-103.

29. Cheng CY, Hong CJ, Yu YW, et al. Brain-derived neurotrophic factor (Val66Met) genetic polymorphism is associated with substance abuse in males. Brain Res Mol Brain Res. 2005;140(1-2):86-90.

30. Bawor M, Brittany BD, Charlie T, et al. Contribution of BDNF and DRD2 genetic polymorphisms to continued opioid use in patients receiving methadone treatment for opioid use disorder: an observational study. Addict Sci Clin Pract. 2015;10:19.

31. Tsai S, Hong CJ, Yu YW, et al. Association study of brain-derived neurotrophic factor (BDNF) Val66Met polymorphism and personality trait and intelligence in healthy young females. Neuropsychobiology. 2004;49(1):13-16.

32. Rajagopal L, Bill W Massey, Michael E, et al. Serotonin (5-HT)1A receptor agonism and 5-HT7 receptor antagonism ameliorate the subchronic phencyclidine-induced deficit in executive functioning in mice. Psychopharmacology. 2016;233(4):649-660.

33. Rajagopal L, Bill W Massey, Mei Huang, et al. Pharmacological data from the novel object recognition (NOR) test: significance and clinical relevance for treatment of cognitive deficits in schizophrenia. Curr Pharm Des. 2014;20(31):5104-5114.

34. Meltzer HY, Horiguchi M, Massey BW. The Role of Serotonin in the NMDA Receptor Antagonist Models of Psychosis and Cognitive Impairment. Psychopharmacology. 2011;213(2-3):289-305.

35. Haerian BS, Haerian MS. OPRM1 rs1799971 polymorphism and opioid dependence: evidence from a meta-analysis. Pharmacogenomics. 2013;14(7):813-824.

36. Schwantes-An T, Zhang J, Chen LS, et al. Association of the OPRM1 Variant rs1799971 (A118G) with Non-Specific Liability to Substance Dependence in a Collaborative de novo Meta-Analysis of European-Ancestry Cohorts. Behav Genet. 2016;46(2):151-169. 
37. Oroszi G, Raymond F, Omalley S, et al. OPRM1 Asn40Asp predicts response to naltrexone treatment; a haplotype-based approach. Alcohol Clin Exp Res. 2009;33(3):383-393.

38. Bauer IE, Soares JC, Nielsen DA. The role of opioidergic genes in the treatment outcome of drug addiction pharmacotherapy: A systematic review. Am J Addict. 2015;24(1):15-23.

39. Drakenberg K, Andrej N, Fagergren P, et al. Mu opioid receptor polymorphism in association with striatal opioid neuropeptide gene expression in heroin abusers. Proc Natl Acad Sci. 2006;103(20):7883-7888.

40. Margolis EB, Hjelmstad GO, Fujita W, et al. Direct bidirectional mu-opioid control of midbrain dopamine neurons. J Neurosci. 2014;34(44):14707-14716.

41. Gianoulakis C. Endogenous opioids and addiction to alcohol and other drugs of abuse. Curr Top Med Chem . 2004;4(1):39-50.

42. Gerfen CR, Engber TM, Mahan LC, et al. D1 and D2 dopamine receptor-regulated gene expression of striatonigral and striatopallidal neurons. Science. 1990;250(4986):1429-1432.

43. Le Merrer J, Becker JA, Befort K, et al. Reward processing by the opioid system in the brain. Physiol Rev. 2009;89(4):1379-1412.

44. Koob GF, Buck CL, Cohen A, et al. Addiction as a stress surfeit disorder. Neuropharmacology. 2014;76:370-382.

45. Domino EF, Hirasawa-Fujita M, Ni L, et al. Regional brain [(11)C] carfentanil binding following tobacco smoking. Prog Neuropsychopharmacol Biol Psychiatry. 2015;59:100-104.

46. Ehrich JM, Phillips PE, Chavkin C. Kappa opioid receptor activation potentiates the cocaine-induced increase in evoked dopamine release recorded in vivo in the mouse nucleus accumbens. Neuropsychopharmacology. 2014;39(13):3036-3048.

47. Tejeda HA, Natividad LA, Orfila JE, et al. Dysregulation of kappa-opioid receptor systems by chronic nicotine modulate the nicotine withdrawal syndrome in an age-dependent manner. Psychopharmacology. 2012;224(2):289-301

48. Grosshans M, Mutschler J, Kiefer F. Treatment of cocaine craving with as-needed nalmefene, a partial $\mathrm{k}$ opioid receptor agonist: first clinical experience. Int Clin Psychopharmacol. 2015;30(4):237-238.

49. Carroll M, Meisch R. Increased drug-reinforced behavior due to food-deprivation 1984. Advances in Behavioral Pharmacology. $1984 ; 4: 47-88$.

50. Nader MA, Woolverton WL. Choice between cocaine and food by rhesus monkeys: effects of conditions of food availability. Behav Pharmacol. 1992;3(6):635-638.
51. Baile CA, Herrera MG, Mayer J. Ventromedial hypothalamus and hyperphagia in hyperglycemic obese mice. Am $J$ Physiol. 1970;218(3):857-863.

52. Bickerdike MJ. 5-HT2C Receptor Agonists as Potential Drugs for the Treatment of Obesity. Curr Top Med Chem. 2003;3(8):885-897.

53. Neelakantan H, Holliday ED, Fox RG, et al. Lorcaserin suppresses oxycodone self-administration and relapse vulnerability in rats. ACS Chem Neurosci. 2017;8(5):1065-1073.

54. Meltzer HY, Massey BW. The role of serotonin receptors in the action of atypical antipsychotic drugs. Curr Opin Pharmacol. 2011;11(1):59-67.

55. Meltzer HY, Massey BW, Horiguchi M. Serotonin receptors as targets for drugs useful to treat psychosis and cognitive impairment in schizophrenia. Curr Pharm Biotechnol. 2012;13(8):1572-1586.

56. Lynch WJ, Peterson AB, Sanchez V, et al. Exercise as a Novel Treatment for Drug Addiction: A Neurobiological and Stage-Dependent Hypothesis. Neurosci Biobehav Rev. 2013;37(8):1622-1644.

57. Sacrey LA, Travis SG, Whishaw IQ. Drug treatment and familiar music aids an attention shift from vision to somatosensation in Parkinson's disease on the reach-to-eat task. Behav Brain Res. 2011;217(2):391-398.

58. Schiavio A, Altenmuller E. Exploring music-based rehabilitation for parkinsonism through embodied cognitive science. Front Neurol. 2015;6:217.

59. Bickel W, Alexandra M, Sarah E, et al. Novel therapeutics for addiction: Behavioral economic and neuroeconomic approaches. Curr treat Options Psych. 2016;3(3):277-292.

60. Pendergast M, Podus D, Finney J, et al. Contingency management for treatment of substance use disorders: a meta-analysis. Addiction. 2006;101(11):1546-1560

61. Terraneo A, Leggio L, Saladini M, et al. Transcranial magnetic stimulation of dorsolateral prefrontal cortex reduces cocaine use: a pilot study. Neuropsychopharmacol. 2016;26(1):37-44.

62. Klingberg T. Training and plasticity of working memory. Trends Cogn Sci. 2010;14(7):317-324.

63. Schmaal L, Goudriaan AE, Joos L, et al. Neural substrates of impulsive decision making modulated by modafinil in alcohol-dependent subjects. Psychol Med. 2014;44(13):2787-2798.

64. Bluth MH, Pincus MR. Narcotic analgesics and common drugs of abuse: Clinical correlations and laboratory assessment. Clin Lab Med. 2016;36(4):603-634.

65. Schwartz DA, George MP, Bluth MH. Toxicology in addiction medicine. Clin Lab Med. 2016;36(4):685-692. 